\title{
Percepciones de los estudiantes de español sobre la creación de un curso para la carrera de comunicación
}

\author{
Edgar Mauricio Vargas Blanco ${ }^{1} \cdot$ Kenny Montgomery \\ New Mexico Highlands University \\ College of Liberal Arts, Departments of Languages and Culture
}

\section{Article history}

Paper received: 21 Sept 2019 Paper received in revised form and accepted for publication: 21 March 2019

\section{Keywords}

linguistic motivations; Spanish for the professions; qualitative and quantitative methods; curriculum; Houston

\section{Palabras clave}

motivaciones lingüísticas; español de las profesiones; métodos cualitativos y cuantitativos; currículo; Houston

\section{ABSTRACT}

The following study delves into perceptions of students on the topic of the creation of courses in Spanish for specific purposes (SSP) or Spanish for the professions, specifically for the Communication degree at the University of Houston in the United States. With the use of quantitative and qualitative methods, 60 Communication students were interviewed to recognize their interest in a course focused on their degree and its contents. The results showed that $88 \%$ of the participants were interested in taking SSP courses because perceive it as a necessity in the city of Houston and in the United States. Their principal motivations are: to improve work opportunities, obtain credits for their degree, and communicate with Hispanics in the city. In regards to the course content, the respondents declared that they wanted to concentrate on vocabulary based around what they study, know the cultural details related to their communication degree and have access to exercises to improve their interpersonal skills.

\section{RESUMEN}

El siguiente estudio describe las percepciones de los estudiantes de cursos de español como lengua extranjera sobre la creación de cursos de español con fines específicos $(\mathrm{EFE})^{2}$ o español para las profesiones, específicamente para la carrera de comunicación en la Universidad de Houston en los Estados Unidos. Con el uso de métodos cualitativos y cualitativos se entrevistaron 60 estudiantes del área de comunicación para reconocer el interés de los participantes en un curso enfocado en su carrera y sobre los contenidos de este. Los resultados mostraron que un $88 \%$ de los participants están interesados en tomar cursos de EFE porque lo ven como una necesidad en la

1 Corresponding author· emvargas@nmhu.edu · (1) https://orcid.org/0000-0001-6249-7348

2 La abreviación EFE se usa durante todo el texto para hacer referencia al español con fines específicos. 
ciudad de Houston y en Los Estados Unidos. Dentro de sus motivaciones principales están: mejorar oportunidades de trabajo, obtener créditos para su carrera y comunicarse con hispanos en la ciudad. Con respecto al contenido del curso, los encuestados afirmaron que desean recibir un vocabulario enfocado en lo que estudian, conocer los detalles culturales relacionados con la carrera y tener acceso a ejercicios donde mejoren sus habilidades interpersonales.

\section{Introduction}

El crecimiento de los hispanos en los Estados Unidos ha sido más fuerte en los últimos años. Beaudrie y Fairclough (2012) reportan que solo en una década esta población se ha duplicado y se espera que en el año 2050 llegue a ser el 30\% de los EE.UU. (aproximadamente 132,8 millones de personas). Por lo tanto, se ha notado el interés por esta minoría en varios ámbitos como la publicidad, la educación, la música la medicina y el idioma. La lengua española es la más estudiada en los EE.UU. alcanzando a tener un porcentaje mayor al $50 \%$ del total de todas las lenguas extranjeras que se ofrecen en las instituciones educativas universitarias (Alonso, 2000). Por esta razón en varias universidades se ha creado un departamento de español separado de los otros idiomas. La Universidad de Houston no se queda atrás frente a este crecimiento al establecer su propio Departamento de Estudios Hispanos durante los años 2007 y 2008.

Sin embargo, en los últimos años se ha visto la necesidad de crear cursos en los que no solo se aprenda sobre la lengua, sino un lenguaje destinado a profesiones específicas. Dentro de las carreras de la universidad que toman español como lengua extranjera para cumplir sus requisitos se encuentra el ámbito de las comunicaciones y es una de las carreras profesionales que cuenta con más alumnos en el programa. El siguiente estudio se enfoca en determinar las percepciones de los estudiantes de la carrera de comunicación de la Universidad de Houston sobre el diseño de un curso en español enfocado a sus necesidades profesionales.

\section{RESEÑA TEÓRICA E INVESTIGATIVA}

Los cursos especializados de EFE se han creado para la enseñanza de conocimientos lingüísticos especializados en diferentes lenguas. Según Gómez (2001) estos lenguajes son una variedad funcional del idioma común y poseen características habituales con respecto a las estructuras gramaticales en todos los niveles lingüísticos. Además, gozan de rasgos parecidos ya que cuentan con un número concreto de beneficiarios que usan este tipo de lenguaje profesional que es usado en situaciones particulares de comunicación (Vendryes, 1929).

Lafford (2013) organizó los siguientes beneficios de tomar clases en EFE: "un claro entendimiento de la interacción de las características del aprendiz y los rasgos contextuales durante la adquisición de la lengua, los procesos de adquisición en escenarios reales, la adquisición de competencia comunicativa intercultural en lugares profesionales y las aplicaciones pedagógicas de visiones de este tipo de 
investigación para clases regulares y de EFE en las que los aprendices se preparan para un aprendizaje a largo plazo de la lenguas meta" (83).

Long y Uscinski (2012) indican que poco a poco se han logrado incorporar los cursos para las profesiones en los programas de las universidades y que este campo se ha consolidado como otra opción diferente a la lingüística, los estudios culturales y la literatura. Además, señalan que este tipo de programas se ofrecen en instituciones medianas o grandes con unos 5000 a 30000 estudiantes.

Lafford, Abbott y Lear (2014) indican la necesidad de investigaciones en otras lenguas ya que la mayoría de los estudios de lenguas para fines específicos son elaborados en inglés y además informan acerca de la falta de profesores entrenados adecuadamente para este tipo de clases. Es poco lo que se ha investigado con respecto al EFE. Upton (2012) señala que investigadores de lengua para fines específicos en lenguas como el español no cuentan con una robusta infraestructura de investigación para apoyar sus estudios. Long (2017) comenta que recientemente se ha discutido como estimular a los estudiantes para aprender lenguas diferentes al inglés porque hay una escasez de graduados que tengan las habilidades de lengua para competir globalmente.

Algunas de las investigaciones se han hecho con respecto a la medicina (De Pheils \& Nadeli, 2009; Lear 2005), los negocios (Abbot \& Lear, 2010; Grosse \& King, 2005) pero el área de la comunicación no ha sido explorada. Es importante conocer más acerca de este campo ya que los hispanos se han convertido en un mercado muy seductor para todo tipo de empresas y en especial se observa el crecimiento de medios de comunicación en español en los Estados Unidos. Silva-Corvalán (2001) señala los resultados de una encuesta a nivel nacional sobre cómo los hispanos gastan su dinero. Casi el 80 por ciento de los hispanos declararon que usan los medios de comunicación en ambas lenguas. La autora presenta que, en los Ángeles, un 55 por ciento y un 60 por ciento de los entrevistados dijeron que prefieren la publicidad en español porque comprenden más la información en este idioma. Ella finaliza expresando que estos resultados inducen a los publicistas y a muchos otros profesionales de la comunicación a buscar maneras de poder llegar a miles de hispanos con su trabajo.

Beaudrie, Ducar y Relaño-Pastor (2009) y Bercher (2006) proponen que las voces de los estudiantes necesitan tener un rol crucial en el programa y en las decisiones del currículo. Tomando en cuenta lo mencionado por estos autores y la importancia de la creación de cursos de EFE sobre la comunicación es que se ha elaborado este trabajo. Es relevante escuchar a las personas que van a recibir la educación en este campo para dirigir de una manera más atractiva la enseñanza de este tipo de cursos. Por lo tanto, las preguntas de investigación que guiaron el análisis de las percepciones de los estudiantes de la carrera de comunicación fueron las siguientes:

1) ¿Estarían los participantes interesados en tomar una clase de EFE?

2) ¿Cuáles serían sus motivaciones?

3) ¿Que contenido y actividades deberían tener este tipo de clases?

\section{LA METODOLOGÍA}

\subsection{La encuesta y la entrevista}


Este estudio se realizó usando métodos cualitativos y cuantitativos. Las encuestas y las entrevistas se elaboraron basadas en los instrumentos diseñados por Chia, Johnson, Chia, y Olive (1999). El cuestionario (ver anexo A) se elaboró con preguntas que los participantes respondieron en el salón de clase y los estudiantes tomaron 20 minutos para llenarlo. Las preguntas buscaron determinar el grado de interés que pueden tener los alumnos de la universidad en tomar una clase de EFE en comunicación después de terminar sus requisitos de lengua. En la primera parte, se enfoca en la información personal de los encuestados como el nombre, la edad y sus especialidades en la universidad. En la segunda parte, las preguntas 1 y 2 se hicieron para determinar si los participantes podrían estar interesados en tomar cursos de EFE y las motivaciones para hacerlo. En las siguientes preguntas se examinaron las percepciones de los estudiantes con respecto al contenido y las actividades que debe tener la clase de EFE.

Este estudio también usa la entrevista como método cualitativo. Vargas Blanco (2018) señala que los métodos cualitativos se usan para comprender mejor las percepciones de los participantes y las razones de sus actitudes hacia la lengua. Las entrevistas se grabaron por 20 minutos con preguntas abiertas en oficinas de la Universidad de Houston. Las conversaciones grabadas fueron informales en las cuales se habló de temas como el interés en una clase, el currículo y las actividades para tener este tipo de clases para fines específicos.

\subsection{Los participantes}

Los participantes fueron seleccionados para completar la encuesta y la entrevista teniendo en cuenta la carrera que estudiaban y el nivel en el que se encontraban. Se escogieron 60 estudiantes que tomaban una clase de segundo año (2301 o 2302) en otoño del año 2015 y la primavera del año 2016. En los resultados de esta investigación, se utilizó la abreviatura $\mathrm{P}$ para hablar de los participantes. Todos los entrevistados estudiaban una de las ramas en las que se pueden especializar en comunicación. No se incluyeron grupos de primer año por su falta de experiencia con el departamento de español ya que solo han tomado las dos primeras clases básicas. Dentro de las diferentes áreas que se pueden estudiar en el departamento de comunicación se encuentran las siguientes: comunicación corporativa, producción de medios de comunicación, comunicación de la salud, periodismo, comunicación interpersonal, relaciones públicas y publicidad. Los datos del estudio se recogieron de varias clases. Los estudiantes completaron la encuesta en clase y otros lo respondieron y lo enviaron de vuelta vía correo electrónico. Todos fueron entrevistados en salones privados de la universidad. Los participantes de este estudio tenían entre 19 y 26 años.

\subsection{La Universidad de Houston}

Esta investigación se realizó en el campus principal de la Universidad de Houston. Esta universidad cuenta con un Departamento de Estudios Hispanos en constante crecimiento. De acuerdo a la Dra. Marta Faiclough, decana interina cuando se creó el departamento, este se separó del Departamento 
de Lenguas Modernas y Clásicas entre los años 2007 y 2008. En el momento existen dos programas para estudiantes que deseen estudiar español a nivel no graduado en los niveles básicos: los cursos de español para lengua extranjera y los de herencia los cuales se realizan en los primeros dos años. Después de estas clases los alumnos pueden continuar estudiando español como especialidad primaria o secundaria. Dentro de los cursos que los estudiantes pueden estudiar en EFE se encuentran los siguientes: Español para las profesiones globales y Contextos de negocios a través de culturas: Estados Unidos y Latinoamérica.

\section{LOS RESULTADOS}

Después de obtener los resultados de las encuestas y las entrevistas, se analizaron las respuestas de los participantes en el estudio. A continuación, se van a presentar los resultados que se obtuvieron. Como los estudiantes tuvieron la oportunidad de escribir sus respuestas o de contestar libremente, es posible que varios hayan señalado varias opciones y por lo tanto al contabilizar el número de estudiantes nos da un porcentaje que puede ser mayor al cien por ciento en algunos casos.

\subsection{Interés por tomar una clase de EFE}

En esta parte de este estudio se buscó reconocer si los estudiantes estarían interesados en tomar una clase de EFE para la comunicación. Como se puede apreciar en la Cuadro 1, la mayoría de los estudiantes tomarían la clase por razones como las siguientes: añadir oportunidades de trabajo, ser bilingües o simplemente para comunicarse en otros países. Los participantes reconocieron algunos de los beneficios de tomar clase de EFE descritos por Lafford (2013) tales como: la adquisición de la competencia comunicativa intercultural y los procesos de adquisición en escenarios reales.

De la misma manera que Silva Corvalán (2001), los participantes hicieron referencia a la importancia económica de la comunidad latina especialmente en la publicidad y en los medios de comunicación en EE.UU. En el siguiente fragmento una de las participantes revela los intereses de tomar este tipo de clase y su relevancia a nivel económico:

$$
\begin{aligned}
& \text { I'd love to take a class like that...however the university does not offer anything like that... Spanish, } \\
& \text { in the US and in this city, is necessary and I want to learn more vocabulary related to my degree... } \\
& \text { There are many companies here that require people with experience in the field and this class } \\
& \text { would be perfect for that...We have a lot of radio stations and TV channels that have the money to } \\
& \text { change things. (P32) }
\end{aligned}
$$

Al igual que en el estudio de Chia et al. (1999), los estudiantes señalaron que la lengua les aportaría beneficios para sus carreras. Sin embargo, los participantes de este estudio comentaron que existe una amplia demanda de personas bilingües en español e inglés en donde viven.

Me gustaría tomar una clase como esa... sin embargo la universidad no la ofrece...el español, en los EE.UU. y en la ciudad, es necesario y quiero aprender más vocabulario relacionado con mi carrera...hay muchas compañías aquí que requieren gente con experiencia en el campo y esta clase seria perfecta para eso...Tenemos muchas estaciones de radio y canales de televisión y ellos mueven mucho dinero. 


\begin{tabular}{|c|c|c|}
\hline ¿Tomaría la clase? & Porcentaje & Comentarios \\
\hline Sí & $88 \%$ & $\begin{array}{l}\text { - Para poder tener más oportunidades de trabajo. } \\
\text { - } \quad \text { Expandir mi red profesional y cultural } \\
\text { - } \quad \text { graduarme. } \\
\text { - Si cuenta como parte de mi especialización principal. } \\
\text { - Para saber cómo es la comunicación en otros países. } \\
\text { - Entrenarse en la cultura y negocios globales. } \\
\text { - } \quad \text { Quiero ser bilingüe. } \\
\text { - Necesito saber cómo hablar el idioma para mi trabajo. }\end{array}$ \\
\hline No & $12 \%$ & $\begin{array}{l}\text { - } \quad \text { Estos cursos me bajan el promedio. } \\
\text { - } \quad \text { No me siento preparado para hacerlo. }\end{array}$ \\
\hline
\end{tabular}

Tabla 1. Interés de los participantes por estudiar una clase de EFE.

El $12 \%$ de las personas no estudiarían la clase porque piensan que les bajaría el promedio de sus notas o no se sienten preparados para ese desafío. Por otra parte, todos explicaron que les gustaría tener facilidad para aprender español para poder estudiarlo a fondo y explorar temas relacionados a su carrera en este idioma.

\subsection{La motivación}

Con referencia a la motivación, Gardner (1985) señala que hay dos tipos de motivación. Una que es la motivación integrativa en la que lo hablantes desean aprender la lengua extranjera para asociarse con gente que usa la lengua. Por otro lado, este autor también señala la motivación instrumental que se refiere al valor práctico asociado con la adquisición de la L2: incrementar oportunidades en el trabajo o en negocios, cumplir un requisito y pasar un curso. En la Cuadro 2 se pueden ver los resultados con respecto a este tema.

\begin{tabular}{|c|c|c|}
\hline Tipo de motivación & Porcentaje & Comentarios \\
\hline $\begin{array}{l}\text { Mayores oportunidades en el } \\
\text { trabajo }\end{array}$ & $65 \%$ & $\begin{array}{l}\text { - Muchos trabajos quieren tener personas } \\
\text { bilingües. } \\
\text { - } \quad \text { Adquirir una clientela más amplia. } \\
\text { - Mis compañeros de trabajo hablan español. }\end{array}$ \\
\hline $\begin{array}{l}\text { Para graduarse u obtener } \\
\text { créditos }\end{array}$ & $27 \%$ & $\begin{array}{l}\text { - Lo necesito para mi carrera. } \\
\text { - Obtener créditos en español y en mi primera } \\
\text { especialización. }\end{array}$ \\
\hline Comunicarse con otros & $22 \%$ & $\begin{array}{l}\text { - } \quad \text { Poder hablar con fluidez. } \\
\text { - Interactuar con otros en diferentes contextos. }\end{array}$ \\
\hline Continuar viviendo en Texas & $19 \%$ & $\begin{array}{l}\text { - Hay muchos hispanos en las ciudades. } \\
\text { - Existen muchos anuncios, periódicos, y } \\
\text { agencias latinas en Houston. }\end{array}$ \\
\hline Viajar a otros países & $14 \%$ & - Ir a otros países y obtener créditos. \\
\hline
\end{tabular}




\begin{tabular}{l|l|ll}
\hline & & - Entablar negocios en el extranjero. \\
\hline Otros & $10 \%$ & $\begin{array}{l}\bullet \\
\text { - }\end{array}$ & Soder traducir en la lengua. \\
& &
\end{tabular}

Tabla 2. La motivación para tomar una clase de EFE.

Los estudiantes presentaron una motivación instrumental principalmente. Un 65\% encuentran que su mayor motivación para tomar la clase es la necesidad de los trabajos por personas bilingües, la obtención de una clientela más variada y el poder comunicarse con compañeros de trabajo que hablan español. Una de las participantes se refirió a su motivación por este tipo de clases:
I have a lot of motivation towards learning Spanish, there are a lot of Latinos here. They are everywhere in the city. I have some friends from Mexico and Puerto Rico... I applied for an internship at Univision, but I didn't get it and I think it was because my Spanish was not strong enough. (P4) ${ }^{4}$

Un $27 \%$ de los encuestados expresaron que estudiarían una clase en español para su carrera si se obtuvieran créditos en español y comunicación. Un $22 \%$ señalaron que lo tomarían simplemente para comunicarse con los otros de mejor manera y obtener mayor fluidez en la lengua.

Por otro lado, un 19\% de los participantes tienen una motivación integrativa. Ellos declararon que si van a seguir viviendo en Texas es necesario hablar el español por la población que vive en el estado. Un $14 \%$ de los sujetos quisieran tomar estas clases para realizar viajes a países hispanos y trabajar por un poco de tiempo en alguna empresa en donde puedan aprender sobre los medios de comunicación locales $u$ otros aspectos relacionados con sus estudios.

\subsection{El contenido de la clase}

Los estudiantes respondieron las preguntas 3 y 4 en las que se indagó sobre el tipo de contenido que la clase de EFE debería ofrecer. Beaudrie et al. (2009) y Belcher (2006) proponen que los estudiantes precisan tener un rol en el desarrollo del currículo. Al respecto, seis de los participantes mencionaron que quisieran tener alguna decisión sobre el contenido de las clases que van a tomar. En el siguiente fragmento uno de los estudiantes se refiere a este tema:

(3)

It's interesting that you're asking this question because I want to have options that can benefit what is important to me...I don't have much of a choice but to take the compulsory classes...There should be a survey for students to give suggestions about what we want and need to learn in class. $(\mathrm{P} 24)^{5}$

$4 \quad$ Tengo mucha motivación para aprender español, hay muchos latinos aquí. Están en todas partes en la ciudad. Tengo muchos amigos de México y Puerto Rico...Solicité para una pasantía en Univisión, pero no me la dieron y pienso que fue porque mi español no era lo suficientemente bueno.

$5 \quad$ Es interesante que usted me pregunte esto porque quiero tener opciones que me puedan beneficiar lo que es importante para mí... no tengo muchas opciones más que tomar las clases que son obligatorias...debería existir una encuesta para que los estudiantes den sugerencias acerca de lo que queremos y necesitamos aprender en las clases. 
Por otro lado, como se puede ver en la Cuadro 3, el $27 \%$ de los estudiantes indicaron que el contenido de la clase debería enfocarse en un vocabulario relevante a la carrera y que se usa diariamente. Sin embargo, desean conocer las expresiones populares que se usan en los países hispanos y el vocabulario relacionado con lo que estudian. El 38\% de los participantes señalaron que el contenido cultural era importante en el desarrollo de la clase, especialmente explicando como los negocios funcionan en los países hispanos, la información cultural reciente e importante y otros aspectos en los cuales ellos puedan conocer las similitudes y diferencias entre las dos culturas.

Las habilidades interpersonales y de habla solo fueron el $26 \%$. Para algunos, la escritura es algo secundario y expresan el deseo por un enfoque hacia la comunicación oral básica y relaciones con personas hispanas. En el 15\% final de los resultados, los participantes indicaron que se debería acortar el contenido de los cursos porque es mucho lo que se abarca y desean que se profundice en clase los temas. Otros participantes señalaron que quisieran aprender cómo escribir en pocas palabras memos o correos electrónicos para comunicarse brevemente y con claridad.

\begin{tabular}{|c|c|c|}
\hline Contenido & Porcentaje & Comentarios \\
\hline Vocabulario & $27 \%$ & $\begin{array}{l}\text { - Vocabulario que se usa en el día a día. } \\
\text { - Términos de la carrera específicos. } \\
\text { - Más expresiones y jerga en el campo de } \\
\text { estudio. }\end{array}$ \\
\hline Cultura & $38 \%$ & $\begin{array}{l}\text { - La manera como las empresas de } \\
\text { comunicación operan en otros países. } \\
\text { - Eventos recientes y relevantes. } \\
\text { - } \quad \text { Debería ser el foco principal. } \\
\text { - ha historia de los medios de comunicación } \\
\text { hispanos. }\end{array}$ \\
\hline $\begin{array}{l}\text { Habilidades } \\
\text { interpersonales y de } \\
\text { habla }\end{array}$ & $26 \%$ & $\begin{array}{l}\text { - } \quad \text { Cómo comunicarse efectivamente. } \\
\text { - Más habla que escritura. } \\
\text { - Temas de comunicación básicos. } \\
\text { - Situaciones en vídeo. }\end{array}$ \\
\hline Otros & $15 \%$ & $\begin{array}{l}\text { - Menos contenido para la clase. } \\
\text { - No hay nada relacionado en español para mi } \\
\text { carrera. } \\
\text { - Escribir simple y al punto. }\end{array}$ \\
\hline
\end{tabular}

Tabla 3. El contenido de la clase de EFE.

\subsection{Las actividades de la clase}

En las preguntas 5 y 6 se examinaron el tipo de actividades que un curso de EFE debería tener. En general, los participantes ofrecieron diferentes respuestas. Como se puede apreciar en la Cuadro 4, un $32 \%$ de los participantes desearían actividades grupales en la que pudieran conocer más gente y donde se elaboraran proyectos de aula como la creación de una corporación ficticia. Los participantes apuntaron que las actividades grupales eran beneficiosas ya que aprendían mutuamente de sus compañeros. Asimismo, señalaron que les gustaría hacer trabajos de investigación sobre gente o empresas locales o internacionales. 


\begin{tabular}{|c|c|c|}
\hline Tipo de actividad & Porcentajes & Comentarios \\
\hline Actividad en grupos & $32 \%$ & $\begin{array}{l}\text { - Crear una corporación falsa y presentarla a la } \\
\text { clase. } \\
\text { - Investigación con varios compañeros. }\end{array}$ \\
\hline Presentaciones & $25 \%$ & $\begin{array}{l}\text { - Pequeño discurso cada semana. } \\
\text { - Hablar sobre problemas de la salud. }\end{array}$ \\
\hline Videos o ejercicios de escucha & $22 \%$ & $\begin{array}{l}\text { - Escuchar Univisión, Telemundo u otro show. } \\
\text { - Mirar películas. }\end{array}$ \\
\hline Talleres de escritura & $19 \%$ & $\begin{array}{l}\text { - } \quad \text { Redactar noticias cortas. } \\
\text { - } \quad \text { Hacer memos o discursos de venta. } \\
\text { - } \quad \text { Para retener información vocabulario y } \\
\text { estructura de las oraciones. }\end{array}$ \\
\hline Fuera de la clase & $22 \%$ & $\begin{array}{ll}\text { - } & \text { Visitar un negocio. } \\
\text { - } & \text { Ferias culturales. } \\
\text { - } & \text { Conducir una campaña con negocios locales. } \\
\text { - } & \text { Hacer investigaciones para obtener datos. } \\
\text { - } & \text { Aprendizaje-servicio (APS). }\end{array}$ \\
\hline $\begin{array}{l}\text { Videos grabados por los } \\
\text { estudiantes }\end{array}$ & $15 \%$ & $\begin{array}{l}\text { En lugar de grabarlo con un compañero, } \\
\text { mejor con alguien nativo que trabaje en el } \\
\text { área de la comunicación. }\end{array}$ \\
\hline Culturales & $27 \%$ & $\begin{array}{l}\text { - Conocer lo que los latinos han traído al } \\
\text { campo de la comunicación. } \\
\text { Diferencia entre las técnicas de investigación } \\
\text { de los anuncios. }\end{array}$ \\
\hline Otros & $25 \%$ & $\begin{array}{l}\text { - Más actividades orales. } \\
\text { - Juegos relacionados con la carrera. } \\
\text { - Mezclar varios medios de comunicación. } \\
\text { - Conversaciones orales } 1 \text { a } 1 \text { sobre los medios } \\
\text { - } \quad \text { de comunicación. } \\
\text { en el mundo hispano. }\end{array}$ \\
\hline
\end{tabular}

Tabla 4. Las actividades de la clase de EFE.

Como se observa en la Cuadro 4, un 25\% de los estudiantes creen importantes actividades como las presentaciones (para poder hablar más y perder el miedo), videos o ejercicios de escucha (grabaciones sacadas de programas hispanos), talleres de escritura (escribir noticias cortas y otro tipo de escritos periodísticos) y actividades fuera de la clase (visitar negocios o empresas relacionadas con el periodismo). EL 22\% de los encuestados reportaron que quisieran tener algunas actividades fuera del aula como visitar compañías o ir a ferias culturales. Al igual que Mary K. Long (2017), dos participantes hablan de la necesidad de aprender una lengua extranjera (en este caso el español) por la globalización y añadieron que la mejor forma seria haciéndolo con aprendizaje-servicio. Uno de los estudiantes se refiere al tema en el siguiente párrafo: 
some community service within the Latino population if possible... it might help me to be more marketable in this globalized world. (P54)

El 15\% de los participantes escogieron la grabación de videos con nativos como una actividad importante para mejorar su nivel de lengua y actividades culturales que se relacionen con su campo de estudio. Por último, 25\% de los entrevistados señalaron diferentes actividades como: juegos, conversaciones orales y forma de comunicación en el mundo hispano. Dentro del grupo de participantes, cinco opinaron no solo de las actividades que querían, sino también de la persona que debería dictar este tipo de cursos:

I want more interesting activities... I would like to speak and use the language in context...however I think we also need well trained professors to be developing these activities for the classes... I would trust a person who has the experience in the field of study. (P45) ${ }^{7}$

Para algunos de los participantes, no solo es relevante el contenido y el tipo de actividades sino que también ven la necesidad de un profesional preparado en estas áreas y que conozca bien de los temas de la carrera.

\section{CONCLUSIONES}

En este estudio se investigaron las actitudes de los estudiantes con respecto a tomar un curso de EFE enfocado en la carrera de la comunicación y su contenido. Se reconoció al vocabulario y la parte cultural como parte esencial que debería ser parte del contenido de sus clases para poder reconocer los contrastes de las culturas hispanas y anglo. Por otra parte, los participantes informaron que desean tener más actividades grupales para investigar afuera sobre empresas relacionadas con su carrera y relacionarse con otros estudiantes. La mayoría de los entrevistados identificó que el español es necesario para su trabajo ya que viven en una ciudad diversa con gran cantidad de personas que hablan español. Unos de los participantes reportaron que, con la necesidad del español en el momento, el departamento podría tener muchos más alumnos, pero se necesita una mejor publicidad y anuncios que motiven a la población de la universidad a tomar sus clases y sobretodo se necesita crear un curso relacionado con las comunicaciones ya que estos son los estudiantes que más toman las clases básicas.

Esta investigación provee las siguientes aportaciones al campo del español con fines específicos. En una primera instancia, se comprueba que la mayoría de los estudiantes desean tomar clases relacionadas de EFE en el área de la comunicación porque lo ven como un beneficio profesional en el

$6 \quad$ Participar en actividades dentro y fuera de la clase son importantes. Estamos compitiendo con una gran comunidad latina en Houston y la mayoría de nosotros escasamente hablamos una lengua...quisiera hacer servicio a la comunidad dentro de la población latina si es posible...eso podría ayudarme a venderme más en este mundo globalizado.

7 Quiero más actividades interesantes...me gustaría hablar y usar la lengua en contexto...sin embargo pienso que necesitamos también profesores bien entrenados para desarrollar este tipo de actividades para las clases...confiaría en una persona que tiene la experiencia el campo de estudio. 
lugar donde viven y en este mundo globalizado. Por otro lado, se evidencia que algunos de ellos quieren hacer parte de las decisiones que se toman acerca del currículo y las actividades de la clase, especialmente porque sienten que unas clases no les aportan lo que necesitan para su carrera. Dentro de las sugerencias de los entrevistados en el estudio está la de realizar encuestas sobre el contenido de los cursos antes de que estos se enseñen. Asimismo, se informa sobre la necesidad de profesores preparados y con experiencia para enseñar este tipo de cursos de la manera adecuada. Finalmente, los resultados muestran que los participantes desean tener un currículo variado en el cual no solo se desarrollen actividades en el aula, pero fuera de ella especialmente en actividades de aprendizajeservicio o pasantías en compañías.

\section{LIMITACIONES DEL ESTUDIO}

Como Upton (2012) lo reporta, se necesitan más estudios en el campo de EFE para apoyar estudios como este. Todavía en muchos departamentos de español no existen las clases de EFE y por lo tanto no hay investigadores que desarrollen investigación en esta área. Lafford et al. (2014) señalan que estudios de EFE en algunas universidades no hacen parte del canon para los portafolios para tener una planta permanente. Por otro lado, otra de las limitaciones del estudio fue la de encontrar espacios privados para las grabaciones con los participantes.

\section{About the authors}

Edgar M. Vargas Blanco is an Assistant Professor of Hispanic Linguistics and Director of the Language Learning Center at New Mexico Highlands University. He holds a Ph.D. in Hispanic Linguistics from the University of Houston and has worked as a Spanish and Linguistics instructor in Colombia, France, England, and the United States. He was part of a Fulbright project in Central Asia during summer 2018. His research interests are in sociolinguistics, Applied Linguistics, Heritage language teaching, and Spanish for specific purposes.

Kenny Montgomery holds a Ph.D. in Hispanic Linguistics from the University of Houston and she is a current Foreign Language instructor at New Braunfels ISD. Her field of research deals with Spanish language acquisition and development in the USA. She has been an international and visiting student at the University of Sorbonne in 
Paris, France, and at The University of Laval in Québec, Canada. She has four years of experience as a Spanish and French professor at the university level in South Texas.

\section{References}

Abbot, A. \& Lear, D. (2010) 'Marketing business Languages: Teaching students to value and promote their coursework", Global Business Languages: Vol. 15, Article 2. Disponible en: http//docs.lib.purdue.edu/gbl/vol15/iss1/2

Alonso, C. (2006) "Spanish: The foreign national language." ADFL Bulletin 37.2-3: 15-15-20. Linguistics and Language Behavior Abstracts (LLBA).

Beaudrie, S., Ducar, C., \& Relaño-Pastor, A.M. (2009).Curricular perspectives in the heritage language context: Assessing culture and identity. Language, Culture and Curriculum, 22 (2), 157-174.

Beaudrie, S. \& Fairclough, M. (2012). Spanish as a heritage language in the United States: The state of the field. Washington, DC: Georgetown University Press.

Belcher, D. (2006). "English for specific purposes: Teaching to perceived needs and imagined futures in worlds of work, study, and everyday life." TESOL Quarterly 40 (1): 133-56.

Chia, H.U., Johnson, R., Chia, H.L., \& Olive, F. (1999). English for college students in Taiwan: A study of perceptions of English needs in a medical context. English for Specific Purposes, 18(2), 107-119. https://doi.org/10.1016/S08894906(97)00052-5

Gardner, R. (1985) Social psychology and second language learning: The role of attitudes and motivation. London: Edward Arnold.

Gómez, J. (2001). La enseñanza: Aprendizaje del español con fines específicos. Madrid: Ed. Edinumen.

Pheils, P. B., \& Naledi, M. S. (2009) Communicating with Latino patients. Journal of Nursing Education. September 2009;48(9):515-518. Available from: CINAHL Plus with Full Text, Ipswich, MA.Grose, C. U., \& Voght, G. (1991). The evolution of languages for specific purposes in the United States. The Modern Language Journal 75, 181-195.

Lafford, B.; Abbot, A., \& Lear, D. (2014) Spanish in the professions and in the community in the US. Journal of Spanish Language Teaching 1:2, 171 186, DOI: 10.1080/23247797.2014.970361.

Lear, D. (2005). Spanish for working medical professionals: Linguistic needs. Foreign Language Annals 38: 223-235.

Long, M. K. (2017). Introduction: LSP studies and the creation of translingual and transcultural competence. Language for specific purposes: Trends in curriculum development, Georgetown University Press, Washington, D.C., 2017, pp. 112. JSTOR, www.jstor.org/stable/j.ctt1ps3169.6.

Long, M. K., \& Uscinski, I. (2012). Evolution of languages for specific purposes programs in the United States: 1990-2011. Modern Language Journal 96, 173-189.

Silva-Corvalán, C. (2001). Sociolingüística y pragmática del español. Washington, D.C: Georgetown University Press.

Upton, T. A. (2012). "LSP at 50: Looking Back, Looking Forward." Iberica 23: 9-28.

Van Lier, L. (2004). The ecology and semiotics of language learning. New York: Kluwer Academic. 
Vargas Blanco, E. M. (2018). Actitudes lingüísticas de padres y madres en familias colombianas y mexicanas en Houston: un estudio cualitativo. Forma y Función, [S.I.], v. 31, n.1, p. 155-181, ene. 2018. ISSN 2256-5464 Disponible en: https://revistas.unal.edu.co/index.php/formayfuncion /article/view/70446/64990

Vendryes, J. (1929). Le langage. Introduction linguisticque a l'histoire. Paris: La Renaissance. 


\section{ANEXO}

Anexo A. Encuesta sobre las percepciones de los estudiantes acerca de un curso de EFE

Name Date

Degree: Major

Minor

Classification: Freshman Sophomore Junior Senior

Age

1) If there was a course related to your degree in Spanish, would you take it? Yes No Why?

2) What would be your motivation for taking a class related to your major/minor in Spanish?

3) What kind of content should a class (related to what you study) in Spanish offer?

4) Is there any content that you consider relevant for a communication class in Spanish?

5) What type of activities should this class have?

6) Can you provide examples of activities from the communication classes that you are taking in your major/minor? 